\title{
A FIXED POINT THEOREM FOR CERTAIN OPERATOR VALUED MAPS
}

\author{
D. R. BROWN AND M. J. O'MALLEY'
}

\begin{abstract}
Let $H$ be a real Hilbert space, and let $B_{1}(H)$ denote the space of symmetric, bounded operators on $H$ which have numerical range in $[0,1]$, topologized by the strong operator topology, and let $L$ be a strongly continuous function on $H$ into $B_{1}(H)$. In this paper, methods are given to locate all $z \in H$ which are fixed points of $L$ in the sense that $L(z) z=z$.

In particular, if $w \in H$ and if $\alpha$ and $\beta$ are fixed positive rational numbers with $\alpha \in\left[\frac{1}{2}, \infty\right)$, a decreasing sequence of elements of $B_{1}(H)$ is recursively defined, and converges to $Q \in B_{1}(H)$. If $\alpha>\frac{1}{2}$, then $Q$ is idempotent and $z=Q w$ is a fixed point of $L$, and if $\alpha=\frac{1}{2}, \beta>\frac{1}{2}$, then $z=Q^{\beta_{w}}$ is a fixed point of $L$.
\end{abstract}

1. Introduction. Let $H$ be a real Hilbert space, and let $B_{1}(H)$ denote the space of symmetric, bounded operators on $H$ which have numerical range in $[0,1]$, topologized by the strong operator topology (that is, the topology of point-wise convergence). It is well known [3], that if $T \in B_{1}(H)$, then there exists a unique $S \in B_{1}(H)$ such that $S^{2}=T$. We represent $S$ by $T^{1 / 2}$. The following theorem is due to John Neuberger [2].

Theorem A. Suppose $w \in H, P$ is an orthogonal projection on $H$, and $L$ is a (strongly) continuous function from $H$ into $B_{1}(H)$. Let $Q_{0}=P$, and set $Q_{n+1}=$ $Q_{n}^{1 / 2} L\left(Q_{n}^{1 / 2} w\right) Q_{n}^{1 / 2}, n=0,1,2, \ldots$ Then $\left\{Q_{n}\right\}_{n=0}^{\infty}$ converges to an element $Q \in B_{1}(H)$ for which $z=Q^{1 / 2} w$ is a fixed point of $P$ and a fixed point of $L$ in the sense that $L(z) z=z$.

In this paper, under the same hypotheses as Theorem A, we develop a family of Neuberger-like results to find points $z \in H$ satisfying $L(z) z=z$ and $P(z)=z$. This family includes Neuberger's theorem and has the additional property that "most" of the sequences $\left\{Q_{n}\right\}$ converge to idempotent elements of $B_{1}(H)$. The limit operator of Theorem A need not be idempotent.

Such theorems as those above not only play a valuable role in the search for numerical solutions of partial differential equations, but are also useful, in the finite-dimensional case, in attacking the problem of determining the nonzero fixed points of a function $\phi: R^{n} \rightarrow R^{n}$. In particular, if $x \in R^{n}-$ $\{0\}$, then $x$ is a fixed point of $\phi$ if and only if $A(x) x=x$, where $A$ is the

Received by the editors March 8, 1978 and, in revised form, August 7, 1978.

AMS (MOS) subject classifications (1970). Primary 47B15; Secondary 15A48.

Key words and phrases. Positive operators, fixed points.

'Both authors received partial support under NASA contract NAS-9-15000. 
matrix valued function defined by $A(x)=\left(\|x\|^{-2}\right) \cdot \phi(x) \cdot\left(x^{T}\right)$, where $\phi(x) \cdot$ $\left(x^{T}\right)$ is the matrix product of the column vector $\phi(x)$ with the row vector $x^{T}$. In fact, it follows that $\phi(x)=x, x \neq 0$, if and only if $A(x)$ is a nonzero symmetric idempotent. $^{2}$

2. Fixed points of $L(z)$. Recall that an operator is positive if $\langle A x, x\rangle \geqslant 0$ for all $x \in H$, where $\langle$,$\rangle is the inner product of H$. We presume familiarity with the standard properties of positive operators as set forth, for example, in [3]. By invocation of the spectral theorem, or, alternately, by a sequential construction, it is possible to provide, for any $T \in B_{1}(H)$ and any positive integer $n$, a unique operator $T^{1 / n} \in B_{1}(H)$ such that $\left(T^{1 / n}\right)^{n}=T$. This notion extends immediately to arbitrary positive rational powers of $T$ by defining $T^{r / s}=\left(T^{1 / s}\right)^{r}$. Moreover, by again appealing to the spectral theorem, it follows that if $\left\{Q_{j}\right\}$ is a sequence in $B_{1}(H)$ converging strongly to $Q$, and $t$ is an arbitrary positive rational number, then $\left\{Q_{j}^{t}\right\}$ converges strongly to $Q^{t}$. Finally, recall that the usual quasi-order defined for positive operators by $A \leqslant B$ if and only if $B-A$ is positive satisfies an additional anti-symmetry condition, to wit: if $A$ and $B$ are positive and commute, then $A<B$ and $B \leqslant A$ forces $A=B$.

LEMMA I. Let $Q \in B_{1}(H)$ and let $\alpha$ be a positive rational number other than 1. If $Q^{\alpha}=Q$, then $Q=Q^{2}$; that is, $Q$ is an idempotent.

Proof. Let $\alpha=r / s$; the presumed equality is equivalent to $Q^{r}=Q^{s}$. Without loss of generality, assume $r<s$ and that $r$ is the minimal positive power of $Q$ which reoccurs in the sequence $\left\{Q^{n}\right\}$. From the fact that powers of an operator descend in the quasi-order mentioned above, together with the limited anti-symmetry of this relation, it follows that $Q^{t}=Q^{r}$ for all integral $t$ between $r$ and $s$. From $Q^{r}=Q^{r+1}$, it follows that $Q^{t}=Q^{r}$ for all $t \geqslant r$. If $r$ is odd, then $\left(Q^{(r+1) / 2}\right)^{2}=Q^{r+1}=Q^{2 r}=\left(Q^{r}\right)^{2}$. By uniqueness of square roots, $Q^{r}=Q^{(r+1) / 2}$, whence $r=(r+1) / 2$ and $r=1$. If $r$ is even, then $\left(Q^{r / 2}\right)^{2}=Q^{r}=\left(Q^{r}\right)^{2}$, whence $r=r / 2$, which is impossible for positive $r$. Thus $r=1$ and $Q=Q^{2}$.

We are now ready to prove our

TheOREM 2. Let $w \in H$, let $P$ be an orthogonal projection on $H$, and let $L$ : $H \rightarrow B_{1}(H)$ be strongly continuous. Let $\alpha, \beta$ be positive rational numbers with $\alpha \in\left[\frac{1}{2}, \infty\right)$. Set $Q_{0}=P$, and let $Q_{n+1}=Q_{n}^{\alpha} L\left(Q_{n}^{\beta} w\right) Q_{n}^{\alpha}, n=0,1,2, \ldots$ Then $\left\{Q_{n}\right\}_{n=0}^{\infty}$ is a decreasing sequence of elements of $B_{1}(H)$ which converge to an element $Q \in B_{1}(H)$ such that

(1) if $\alpha>\frac{1}{2}$, then $Q$ is idempotent and $z=Q w$ satisfies $L(z) z=z$, and $P z=z$, and

\footnotetext{
2 It is a pleasure to record our indebtedness to H. P. Decell for the remark immediately above, and to several other members of the University of Houston Mathematics Department, particularly Phillip Walker, for helpful conversations regarding the preparation of this paper.
} 
(2) if $\alpha=\frac{1}{2}$ and $\beta \geqslant \frac{1}{2}$, then $z=Q^{\beta}$ watisfies $L(z) z=z$ and $P z=z$.

Proof. Fix $\alpha \geqslant \frac{1}{2}$ and $\beta>0$. Since $Q_{0}=P \in B_{1}(H)$ and the range of $L$ is in $B_{1}(H)$, it follows inductively that $Q_{n} \in B_{1}(H)$ for all $n$. Since $2 \alpha>1$, $Q_{n}^{2 \alpha} \leqslant Q_{n} ;$ moreover, $\left\langle\left(Q_{n}^{2 \alpha}-Q_{n+1}\right) x, x\right\rangle=\left\langle\left(Q_{n}^{2 \alpha}-Q_{n}^{\alpha} L\left(Q_{n}^{\beta} w\right) Q_{n}^{\alpha}\right) x, x\right\rangle$ $=\left\langle Q_{n}^{\alpha}\left(I-L\left(Q_{n}^{\beta} w\right)\right) Q_{n}^{\alpha} x, x\right\rangle=\left\langle\left(I-L\left(Q_{n}^{\beta} w\right)\right) Q_{n}^{\alpha} x, Q_{n}^{\alpha} x\right\rangle$. Thus, since $I-$ $L\left(Q_{n}^{\beta} w\right) \geqslant 0$, it follows that $Q_{n+1} \leqslant Q_{n}^{2 \alpha}$. Hence we have

$$
Q_{n+1} \leqslant Q_{n}^{2 \alpha} \leqslant Q_{n}, \quad n=0,1,2, \ldots
$$

In particular, the sequence $\left\{Q_{n}\right\}$ is monotonically decreasing in the (operator) interval from 0 to $I$. Thus we have by $\left[3\right.$, p. 318] that the sequence $\left\{Q_{n}\right\}$ converges strongly to an element $Q \in B_{1}(H)$, whence $\left\{Q_{n}^{\alpha}\right\}$ converges to $Q^{\alpha}$ and $\left\{Q_{n}^{\beta}\right\}$ converges to $Q^{\beta}$. Since $L$ is continuous and operator multiplication is jointly continuous in the strong topology on $B_{1}(H)$, we have by uniqueness of limits that $Q=Q^{\alpha} L\left(Q^{\beta} w\right) Q^{\alpha}$. Also, from (*) and the closed graph of the relation $\leqslant$, we have $Q \leqslant Q^{2 \alpha} \leqslant Q$. Thus, since $Q$ and $Q^{2 \alpha}$ commute, we have that $Q=Q^{2 \alpha}$. Moreover, since $P=Q_{0}$, we have $P Q_{n}=Q_{n}$, whence $P Q^{\gamma}=Q^{\gamma}$ for all positive rational $\gamma$.

(i) Suppose $\alpha>\frac{1}{2}$. By Lemma $1, Q=Q^{2}$, from which it follows that $Q=Q^{\gamma}$ for all positive rational $\gamma$, and, in particular, $Q=Q L(Q w) Q$.

Let $z=Q w$, and fix $x \in H$. Then

$$
\langle Q x, x\rangle=\langle Q L(z) Q x, x\rangle=\langle L(z) Q x, Q x\rangle,
$$

and since $Q^{2}=Q$, it follows that

$$
0=\langle Q x, Q x\rangle-\langle L(z) Q x, Q x\rangle=\langle(I-L(z)) Q x, Q x\rangle .
$$

Therefore, since $I-L(z)$ and hence $(I-L(z))^{1 / 2}$ belong to $B_{1}(H)$, we have that $Q=L(z) Q$. In particular, $z=Q w=L(z) Q w=L(z) z$.

(ii) Suppose $\alpha=\frac{1}{2}, \beta \geqslant \frac{1}{2}$. Let $z=Q^{\beta}$; then $Q=Q^{1 / 2} L(z) Q^{1 / 2}$ from which

$$
\langle Q x, x\rangle=\left\langle Q^{1 / 2} L(z) Q^{1 / 2} x, x\right\rangle=\left\langle L(z) Q^{1 / 2} x, Q^{1 / 2} x\right\rangle .
$$

Since $\langle Q x, x\rangle=\left\langle Q^{1 / 2} x, Q^{1 / 2} x\right\rangle$ also, we have

$$
0=\left\langle Q^{1 / 2} x-L(z) Q^{1 / 2} x, Q^{1 / 2} x\right\rangle=\left\langle(I-L(z)) Q^{1 / 2} x, Q^{1 / 2} x\right\rangle .
$$

Now, as in (i), it follows that $Q^{1 / 2}=L(z) Q^{1 / 2}$. In particular,

$$
z=Q^{\beta} w=Q^{1 / 2} Q^{\beta-1 / 2} w=L(z) Q^{1 / 2} Q^{\beta-1 / 2} w=L(z) Q^{\beta} w=L(z) z .
$$

That $P z=z$ in both cases is obvious from the fact that $P Q^{\gamma}=Q^{\gamma}$ for all positive rational $\gamma$. This completes the proof.

Given a nonzero element $z \in H$ such that $L(z) z=z$, it is reasonable to ask if our sequences are able to produce $z$. We note now that, by proper selection of $w$ and $P, z$ is attainable from each of our sequences. Specifically, if $\alpha$ and $\beta$ are fixed as in the theorem, then let $w=z$ and let $P$ be the orthogonal projection of $H$ onto the line through $z$. From the construction of the sequence $\left\{Q_{n}\right\}, Q_{1}=P L(z) P$, whence $Q_{1}=P$. It follows immediately 
that $Q_{n}=P$ for all $n$ and thus $Q=P$. Hence $z=Q w=P w$ (or $z=Q^{\beta} w=$ $\left.P^{\beta} w=P w\right)$ is the fixed point yielded by our theorem.

While it is not reasonable to expect the practitioner to guess $P$ so accurately, these remarks do attach the virtue of theoretical completeness to these processes.

3. Examples. (1) Suppose that $\alpha=\frac{1}{2}$ and that $\gamma, \delta \in\left[\frac{1}{2}, \infty\right)$ such that neither of $\gamma, \delta$ is an integral multiple of the other. We show that for fixed $w \in H$ and $P$, the $Q$ and $z$ obtained by using $\gamma$ for $\beta$ need not be the same as those obtained by using $\delta$ for $\beta$. Moreover, the limit operator $Q$ in this case need not be an indempotent, although it can be one. Assume $\delta<\gamma$. Let $k$ be the least positive integer such that $\gamma<k \delta$. Note $2 \leqslant k$ and $(k-1) \delta<\gamma$. Let $a$ be any number in the interval $(0,1)$. Then $a^{k \delta}<a^{\gamma}<a^{(k-1) \delta} \leqslant a^{\delta}$.

Define $L: R \rightarrow[0,1]$ by

$$
L(x)= \begin{cases}1, & x \leqslant a^{\gamma}, \\ {\left[(1-a) /\left(a^{\gamma}-a^{(k-1) \delta}\right)\right] \cdot\left(x-a^{\gamma}\right)+1,} & a^{\gamma} \leqslant x \leqslant a^{(k-1) \delta}, \\ a, & a^{(k-1) \delta} \leqslant x .\end{cases}
$$

Set $P=1, w=1$. Using $\gamma$ for $\beta$ in the theorem yields $Q_{0}=1$ and $Q_{1}=a$. Inductively, $Q_{n}=a$, so that $Q=a$. Hence $z=Q^{\gamma} w=a^{\gamma} \cdot 1=a^{\gamma}$ in this case. On the other hand, using $\delta$ for $\beta$ gives $Q_{0}=1, Q_{1}=a$, but $Q_{2}=$ $a^{2}, \ldots, Q_{k}=a^{k}$. Moreover, $Q_{n}=a^{k}$ for $n \geqslant k$, hence $Q=a^{k}$ and $z=Q^{\delta} w$ $=a^{k \delta} \cdot 1=a^{k \delta}$. By the choices of $a$ and $k$, the exponents $\gamma$ and $\delta$ yield distinct operators and distinct fixed points. Moreover, neither of the limit operators determined by $\gamma$ and $\delta$ is idempotent.

(2) Suppose that $\alpha>\frac{1}{2}$, so that any limiting $Q$ obtained through the theorem is idempotent. We show for fixed $w \in H$ and $P$, that the resulting limit idempotents may vary with the choice of $\beta$, as may the fixed points determined in this manner. To this end, let $\alpha=1$ in the theorem. Let $L$ : $R^{3} \rightarrow B_{1}\left(R^{3}\right)$ be as follows: all image matrices are diagonal, where

$$
\left(\begin{array}{lll}
x & 0 & 0 \\
0 & y & 0 \\
0 & 0 & z
\end{array}\right)
$$

will be represented as $\operatorname{diag}(x, y, z)$. We require

$$
\begin{array}{rlrl}
L(1,1,1) & =\operatorname{diag}\left(1, \frac{1}{4}, 1\right), & L\left(1, \frac{1}{2}, 1\right) & =\operatorname{diag}\left(1, \frac{1}{4}, \frac{1}{4}\right), \\
L\left(1, \frac{1}{4}, 1\right) & =\operatorname{diag}\left(\frac{1}{4}, \frac{1}{4}, 1\right), \quad L(1, y, z)=\operatorname{diag}(1, y, z)
\end{array}
$$

for $(y, z) \in\left[0, \frac{1}{2}\right] \times\left[0, \frac{1}{2}\right]$, and $L(x, y, 1)=\operatorname{diag}(x, y, 1)$ for $(x, y) \in\left[0, \frac{1}{2}\right] \times\left[0, \frac{1}{2}\right]$. The extension theorem of Tietze (cf. [1]) permits a continuous extension of $L$ to all of $R^{3}$ into the diagonal matrices whose entries are in the interval $[0,1]$. Let $P=I_{3}$, the identity operator, and let $w$ be the vector $(1,1,1)$. If $\beta=\frac{1}{2}$, a brief examination of the defining sequence of $Q_{n}$ 's in Theorem 2 shows that the limit idempotent $Q=\operatorname{diag}(1,0,0)$, and 
$z=Q w=(1,0,0)$. On the other hand, if $\beta=1$, then limit $Q=\operatorname{diag}(0,0,1)$, and $z=(0,0,1)$.

(3) With notation as in (2), suppose $\beta=1$ is fixed. We show for fixed $w \in H$ and $P$, that the resulting limit idempotents may vary with $\alpha$, as may the fixed points determined in this manner. Letting $P=I_{3}$ and $w=(1,1,1)$ as in (2), we require this time that

$$
\begin{gathered}
L(1,1,1)=L\left(1, \frac{1}{2}, 1\right)=\operatorname{diag}\left(1, \frac{1}{2}, 1\right), \\
L\left(1, \frac{1}{8}, 1\right)=L(1,0,0)=\operatorname{diag}(1,0,0), \\
L\left(1, \frac{1}{32}, 1\right)=L(0,0,1)=\operatorname{diag}(0,0,1) .
\end{gathered}
$$

Extending as before, we have a continuous $L$ defined on $R^{3}$ into the diagonal matrices with entries in $[0,1]$. For any choice of $\alpha, Q_{1}=\operatorname{diag}\left(1, \frac{1}{2}, 1\right)$. If $\alpha=1, Q_{2}=\operatorname{diag}\left(1, \frac{1}{8}, 1\right), Q_{3}=Q_{n}=Q=\operatorname{diag}(1,0,0), z=(1,0,0)$. On the other hand, if $\alpha=2$, then $Q_{2}=\operatorname{diag}\left(1, \frac{1}{32}, 1\right), Q_{3}=Q_{n}=Q=\operatorname{diag}(0,0,1)$, $z=(0,0,1)$.

It is easy to see that a slightly more complicated definition of $L$ would yield a single example incorporating the features of all three prior illustrations.

\section{REFERENCES}

1. John L. Kelley, General topology, Van Nostrand, Princeton, N. J., 1955.

2. John Neuberger, Projection methods for linear and nonlinear systems of partial differential equations, Lecture Notes in Math., Springer, Berlin (to appear).

3. Martin Schechter, Principles of functional analysis, Academic Press, New York, 1971.

Department of Mathematics, Universtty of Houston, Houston, TeXas 77004 